\title{
Impulsive stimulated Raman scattering of molecular vibrations using nonlinear pulse shaping
}

\author{
R.A. Bartels *, S. Backus, M.M. Murnane, H.C. Kapteyn \\ Department of Physics, University of Colorado, and JILA, University of Colorado and National Institute of Science and Technology, \\ Boulder, CO 80309-0440, USA
}

Received 14 November 2002; in final form 4 April 2003

\begin{abstract}
We demonstrate the excitation of a molecular vibrational coherence via impulsive stimulated Raman scattering (ISRS) by using a pump pulse longer than the vibrational period of the excited mode. By making use of the reshaping of the pump pulse during nonlinear propagation, a Raman vibrational coherence can be excited via ISRS with a pump pulse incapable of such excitation at the input of the medium.
\end{abstract}

(C) 2003 Elsevier Science B.V. All rights reserved.

When a femtosecond laser pulse propagates through a molecular medium, it excites a molecular coherence in any Raman-active molecular vibrational mode provided the pulse has temporal features faster than the vibrational period. This type of excitation, called impulsive stimulated Raman scattering (ISRS) [1,2], is a general material response and occurs even in the absence of any direct resonant transitions that might be excited by the pulse. To excite a Raman coherence, two colors must be present simultaneously in a pulse whose frequency difference matches the frequency of a vibrational mode. ISRS is possible when a laser pulse possesses a bandwidth broad enough to encompass the entire energy gap from the initial state to the Raman-excited mode. Nevertheless, a

\footnotetext{
${ }^{*}$ Corresponding author. Fax: +1-303-492-5235.

E-mail address: bartels@jila.colorado.edu (R.A. Bartels).
}

Fourier analysis of ISRS shows that pulses with a very large bandwidth will not impulsively excite a Raman coherence unless the pulse possesses temporal structure that is faster than the mode period. For example, if a pulse undergoes pure self-phase modulation (SPM) as it propagates through a medium, its bandwidth can become substantially broadened. However, in the absence of other effects, the pulse envelope will not change. Thus, the self-phase modulated pulse is no more effective than the original pulse at exciting a molecular vibrational coherence.

We demonstrate in this Letter that pulse reshaping of an intense ultrafast pulse propagating through a molecular gas can allow the excitation of a Raman coherence that the unshaped laser pulse was incapable of exciting via ISRS. The unshaped laser pulse is incapable of exciting a coherence because of the absence of fast temporal structure on the pulse and the lack of sufficient 
bandwidth. The pulse reshaping that enables ISRS is a result of self-phase modulation (SPM) coupled with self steepening (SS) under conditions of low dispersion. The SPM broadens the bandwidth, and the SS creates a fast transient on the trailing edge of the laser pulse. When, the trailing edge of the pump pulse becomes faster than the vibrational period of the medium, the pump pulse is capable of exciting a Raman coherence despite the fact that the original pulse was too long for ISRS excitation. These pulse modifications allow an intense ultrashort light pulse to access nonresonant nonlinearities due to this self-evolving behavior of the pulse. Such a self-evolving pulse-shape behavior has important consequences for the propagation of intense femtosecond pulses in the atmosphere $[3,4]$. Furthermore, the decrease in the fall time of the pulse enables the excitation of energetic vibrational modes in molecules using relatively easy to make longer-duration pulses. This access to high frequency, chemically relevant molecular bonds has important ramifications for coherent control of molecular systems [5-8].

In this work, we propagate intense femtosecond laser pulses in a low pressure $(<1 \mathrm{~atm})$ molecular gas contained in a hollow core waveguide. This geometry permits the propagation of an intense femtosecond pulse over a long interaction length in an essentially plane-wave geometry. Hollow core fibers filled with noble gases have been used for high energy ultrafast pulse compression by using self phase modulation [9,10], for efficient phase matching of parametric mixing processes [11], and high harmonic generation [12,13]. When the hollow fiber is filled with a molecular gas, the intense pulse can excite coherent molecular motion. The molecular coherence is time-varying and imposes a temporal phase modulation on a probe pulse. This phase modulation can temporally compress the probe pulse with coherent molecular rotations [14] and vibrations [15,16]. Moreover, spectral sidebands imposed by a vibrational coherence have been used to selectively excite and control multimode vibrational wave packets [6,7]. Our work seeks to extend the utility of these molecular coherent control techniques by controlling the excitation of a Raman coherence in very fast molecular motion that is otherwise inaccessible; we also seek to understand the consequences of pulse self-evolution when new nonlinearities are excited by the modified pump pulse.

The Raman coherence excited by a laser pulse leads to a macroscopic medium polarization. This polarization exists in the medium until a dephasing of the Raman coherence causes the coherence to collapse into a non-equilibrium population distribution. The macroscopic polarization is timevarying and leads to a nonlinear source term in the wave equation which modifies the shape, phase, and spectrum of the laser pulse as it propagates in the molecular medium. In the limit of weak excitation of a Raman coherence, the ISRS process can be described by a simple classical model: the normalized displacement from equilibrium of the nuclear coordinate $Q$ in the impulsive limit can be described by [17]

$\frac{\mathrm{d} Q^{2}}{\mathrm{~d} t^{2}}+2 \gamma \frac{\mathrm{d} Q}{\mathrm{~d} t}+\Omega_{\mathrm{v}}^{2} Q=\frac{1}{2} N\left(\frac{\mathrm{d} \alpha}{\mathrm{d} Q}\right)_{0} E_{\mathrm{L}}^{2}$,

where $\Omega_{\mathrm{v}}$ is the vibrational frequency, $\gamma$ is the dephasing rate of the coherence, $N$ is the number density of oscillators, $(\mathrm{d} \alpha / \mathrm{d} Q)_{0}$ is the change in polarizability with intermolecular distance $Q$, and $E_{\mathrm{L}}^{2}(t)=\frac{1}{4}\left\{2 A_{\mathrm{L}}(t) A_{\mathrm{L}}^{*}(t)+A_{\mathrm{L}}^{2} \mathrm{e}^{-\mathrm{i} 2 \omega_{\mathrm{L}} t}+A_{\mathrm{L}}^{* 2} \mathrm{e}^{\mathrm{i} 2 \omega_{\mathrm{L}} t}\right\}$ is square of the laser field. Note that any induced motion of the nuclei is sensitive only to the laser intensity envelope, $I(t) \propto A_{\mathrm{L}}(t) A_{\mathrm{L}}^{*}(t)$, and not to the electric field directly. The molecular response can be easily calculated using a Green's function analysis for any driving pulse shape $I(t)$, and it shows that the strength of the excited ISRS Raman coherence of a vibrational mode is proportional to the power spectral density of the pulse intensity envelope at that mode's vibrational frequency.

Thus, the shape of the pump pulse intensity determines whether or not a molecular coherence is excited and determines the strength of that coherence. Moreover, this analysis shows that the necessary, sufficient condition for excitation of a vibrational coherence is that the pulse contains structure in the intensity profile that is faster than the vibrational period. The excited vibrational coherence determines the extent to which the molecular response affects the laser pulse during propagation. This is best illustrated by considering two limiting cases of a Raman interaction with a 
single laser pulse. In the ISRS limit, the pulse duration is negligible in comparison to the molecular vibrational period, i.e., $\tau_{\mathrm{p}} \ll 2 \pi / \Omega_{\mathrm{v}}$, and the molecular response is the impulse response of a harmonic oscillator. Therefore, the molecule exhibits damped, sinusoidal oscillations of $Q$ (i.e., molecular vibrations), resulting in a temporal sinusoidal variation in the molecular polarizability. In the adiabatic limit, the pump pulse duration is substantially longer than the vibrational period of the molecule $\left(\tau_{\mathrm{p}} \gg 2 \pi / \Omega_{\mathrm{v}}\right)$, and the molecular displacement follows the pulse intensity, i.e., $Q(t) \propto I(t)$. This stretching modifies the polarizability of the molecule in a way that is indistinguishable from the electronic Kerr response of the molecule in the adiabatic limit. Other, more complex pulse shapes will excite a molecular response that is between these two limits, but a residual molecular vibrational coherence will only be present for pulse shapes with structure faster than the vibrational period. This fast structure implies that the power spectrum of $I(t)$ has non-negligible amplitude at the vibrational frequency.

To understand how the ISRS excitation process can modify the pump pulse during propagation, we consider the nonlinear source term, $\mu_{0} \partial^{2} P_{\mathrm{NL}} / \partial t^{2}$, of the wave equation, which describes how the nonlinear propagation modifies the laser pulse [18]. The nonlinear polarization can be written as a sum of the electronic $\left(P_{\text {ele }}\right)$ and nuclear $\left(P_{\text {nuc }}\right)$ nonlinear polarizations. The electronic nonlinear polarizability is dominated by the Kerr response, $P_{\text {ele }}=\varepsilon_{0} \chi^{(3)}\left|E_{\mathrm{L}}\right|^{2} E_{\mathrm{L}}$, and the nuclear response given by $P_{\text {nuc }}=N(\partial \alpha / \partial Q)_{0} Q E_{\mathrm{L}}$, recall that $Q(t) \propto E_{\mathrm{L}}^{2}$. An expansion of the second derivative of the nonlinear polarizations leads to the following significant terms that oscillate at optical frequencies:

$$
\begin{aligned}
& \left\{-\omega^{2}\left(\frac{3}{4} \frac{\chi^{(3)}}{c^{2}}\left|A_{\mathrm{L}}\right|^{2}+\mu_{0} N\left(\frac{\partial \alpha}{\partial Q}\right) Q(t)\right) A_{\mathrm{L}}\right. \\
& +2 \mathrm{i} \omega \frac{\partial}{\partial t}\left(\frac{3}{4} \frac{\chi^{(3)}}{c^{2}}\left|A_{\mathrm{L}}\right|^{2} A_{\mathrm{L}}\right. \\
& \left.\left.+\mu_{0} N\left(\frac{\partial \alpha}{\partial Q}\right)_{0} Q(t) A_{\mathrm{L}}\right)\right\} \mathrm{e}^{\mathrm{i} \omega t},
\end{aligned}
$$

with the electric field written as $E_{\mathrm{L}}=A_{\mathrm{L}} \mathrm{e}^{\mathrm{i} \omega t}$. The first term, $-\omega^{2}\left(\frac{3}{4} \frac{\chi^{(3)}}{c^{2}}\left|A_{\mathrm{L}}\right|^{2}+\mu_{0} N\left(\frac{\partial \alpha}{\partial Q}\right)_{0} Q(t)\right) A_{\mathrm{L}}$,

results in a temporally varying perturbation to the index of refraction. The resulting temporal phase modulation modifies the power spectrum of the laser pulse, but does not modify the temporal envelope - the excitation of rotational coherences by the pump pulse also imposes a time-dependent phase that modulates the pulse and causes a spectral red-shift [19].

By contrast, the imaginary terms operate on the pump pulse envelope, resulting in a reshaping of the pump pulse temporal profile. The first imaginary term,

$2 \mathrm{i} \frac{3}{4} \frac{\omega \chi^{(3)}}{c^{2}} \frac{\partial}{\partial t}\left(\left|A_{\mathrm{L}}\right|^{2} A_{\mathrm{L}}\right)$,

is self steepening, which slows the group velocity for more intense portions of the laser pulse and leads to the formation of a very fast trailing edge on the laser pulse [18]. If this trailing edge becomes steeper than the vibrational period of a molecular vibrational mode, then a vibrational coherence can be excited by the reshaped pump pulse. The pump pulse self steepening causes a slow increase of the intensity on the leading edge of the pulse that moves the atoms from their equilibrium position on the ground state; then the steep trailing edge 'turns off' on a time scale faster than vibrational period, causing the bond lengths to oscillate. This method can be called displacive ISRS, in contrast to traditional ISRS that imparts an instantaneous change in momentum to the vibrational mode. Displacement from the equilibrium position occurs at later times as a result of that impulsive momentum change.

The other imaginary term can be expanded into two terms that lead to a reshaping of the pump pulse into a train of pulses separated by the vibrational period when a molecular vibrational coherence has been excited. The first such molecular term,

$2 \mathrm{i} \omega \mu_{0} N\left(\frac{\partial \alpha}{\partial Q}\right)_{0} Q(t) \frac{\partial A_{\mathrm{L}}}{\partial t}$,

is analogous to self steepening in that it modifies the local group velocity based on the sign of $Q(t)$, while the second term, 
$2 \mathrm{i} \omega \mu_{0} N\left(\frac{\partial \alpha}{\partial Q}\right)_{0} \frac{\partial Q(t)}{\partial t} A_{\mathrm{L}}$

results in energy loss/gain based on the direction of the local frequency shift imposed by the temporal phase modulation of the molecular response. Both of these molecular envelope terms can result in a break up of the pump pulse into a pulse train with the vibrational period of the molecule; redistribution of the pulse energy in this way causes that pump pulse to more effectively excite a vibrational coherence. The greater the excitation of a molecular vibrational coherence, the larger the magnitude of $Q(t)$ and the more severe the pulse reshaping, which in turn more efficiently excites the vibrational coherence. This positive feedback forms the basis for amplification of the power spectral density in the Fourier transform of $I(t)$ with propagation. To initiate the molecular modulation, the pulse must initially excite a Raman coherence, meaning it must have structure faster than the vibrational period before the ISRS amplification process can begin. This phenomenon also suggests that other spectral broadening and pulse shaping processes might be able to 'seed' the ISRS amplification process and excite coherences in previously inaccessible Raman active modes. In the absence of such a process, a pulse with a duration (or structure) longer than the vibrational period would propagate without exciting a vibrational coherence.

The most significant pulse distortion mechanisms that act to reshape the pump pulse can be identified by considering the characteristic length scales of each interaction. The characteristic length scale for the group velocity dispersion (GVD) is given by $L_{\mathrm{GVD}}=\tau_{\mathrm{p}}^{2} /\left|\beta_{2}\right|$, where $\beta_{2}$ is the GVD. The length scale for SPM and SS are given by $L_{\mathrm{SPM}}=\left(A_{\mathrm{eff}} c / n_{2} \omega_{0} P_{0}\right)$ and $L_{\mathrm{SS}}=\omega_{0} \tau_{\mathrm{p}} L_{\mathrm{SPM}}$, where $n_{2}$ is the nonlinear index, $P_{0}$ is the peak power of the laser pulse, and $A_{\text {eff }}$ is the effective area of the fiber mode [18]. For the experimental conditions used in this work, the GVD length ranges from $\sim 40$ to $350 \mathrm{~m}$, the SPM length is on the order of $1.5-7 \mathrm{~cm}$, while the SS length is on the order of $1-4 \mathrm{~m}$, as compared to the $0.6 \mathrm{~m}$ fiber length. This means that the effects of dispersion are negligible for the initial stage of pulse evolution. (Note that a large increase in bandwidth will make linear dispersion more important.) Since SPM in the presence of negligible dispersion results in essentially no pulse envelope distortion, self steepening will dominate the pulse envelope evolution initially for pump pulses that do not excite a Raman coherence. Homoelle and Gaeta [20] have theoretically studied the propagation of intense femtosecond pulses in argon under similar conditions to those of our experiment. Their calculations show that the pump pulse will develop structure characteristic of self steepening with a slow rising edge and a fast trailing edge.

In our experiment, we use a $1 \mathrm{~mJ}, 1 \mathrm{kHz}$ amplified Ti:sapphire laser system generating 26 fs pulses [21]. A fraction of the pump pulse $(\sim 10 \%)$ is frequency-doubled in a long nonlinear crystal to generate a narrow-bandwidth probe, well-separated spectrally from the pump. The pump and probe pulses are focused into a $60 \mathrm{~cm}$ long, $450 \mu \mathrm{m}$ diameter, hollow-core fiber filled with a molecular gas. The pump pulse excites a vibrational coherence in the molecules [6,7], leading to a time varying index of refraction. Due to the narrow bandwidth of the probe pulse, its duration is much longer than the vibrational period ( 14-24 fs) of the excited coherences in the Raman vibrational modes. When a vibrational coherence is excited, the time-varying index of refraction imposes a periodic, time-dependent phase modulation on probe pulse, scattering energy from the probe spectrum to sidebands. The intensity of the sidebands provides a measure of the level of excitation of the vibrational coherence in the molecular gas.

A typical probe spectrum showing Stokes and anti-Stokes peaks corresponding to excitation of the $\mathrm{CO}_{2}$ asymmetric stretch mode at $38.6 \mathrm{THz}$, and two quanta of the bend mode at $41.6 \mathrm{THz}$ is shown in Fig. 1a, inset. Both of these modes can still be directly excited by the incident pulse. However, as we increase the gas pressure, the light-molecule interaction gets stronger, reshaping the pulse. We can study this by fully characterizing the pump pulse after propagation through the gas, using SHG FROG [22], as shown in Fig. 2. The power spectral density (PSD) of the pulse envelope at the frequency of the Raman vibration is directly proportional to its ability to excite a vibrational 

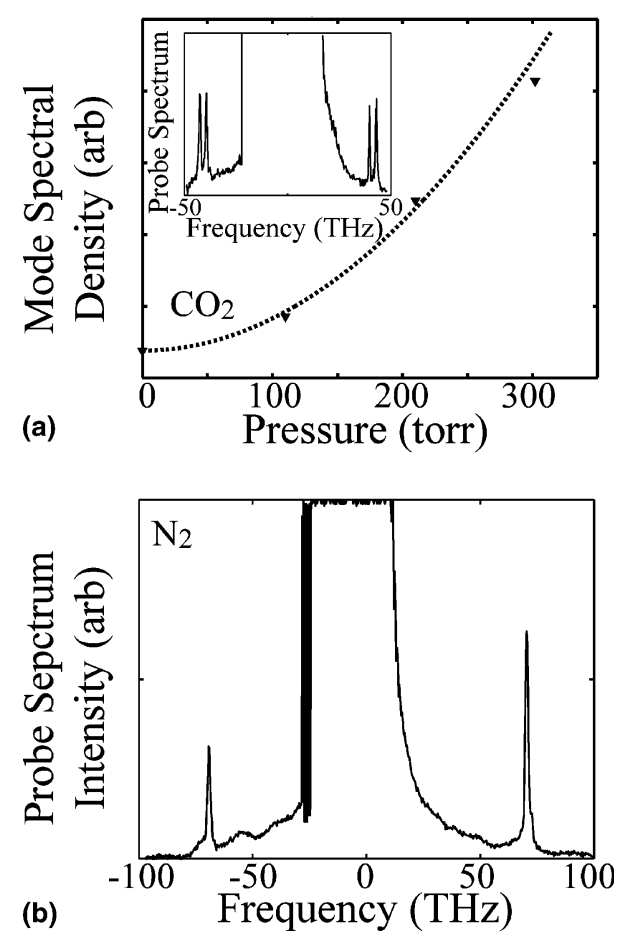

Fig. 1. (a) Power spectral density (Fig. 2, right) at the Raman vibrational frequency of $41.6 \mathrm{THz}$ in $\mathrm{CO}_{2}$ of the measured intensity profile of a laser pulse exiting the $\mathrm{CO}_{2}$ gas, which highlights the increase in the power spectral density at the Raman mode frequency and indicates a development of periodic temporal structure in the pulse at the mode frequency. The inset is a typical probe spectrum for vibrationally excited $\mathrm{CO}_{2}$. (b) The measured probe spectrum in the presence of a vibrational coherence excited nitrogen gas.

coherence. Fig. 1a shows the PSD of the measured intensity profile (Fig. 2) at the $41.6 \mathrm{THz}$ bend mode frequency as a function of pressure. The magnitude of the PSD grows with increasing molecular density. This increase implies an amplification of the temporal structure in the pump pulse capable of exciting vibrational coherences via ISRS.

Although the details of the ISRS amplification are unknown, we clearly observe a 'self-modulation' of the pump pulse leading to an increased excitation of the Raman coherence. For the case of pure SS, the reshaping of the pulse envelope power spectrum would result in a smooth tail that extends to very high frequencies and would not include any periodic structure. The nulls in the $I(t)$
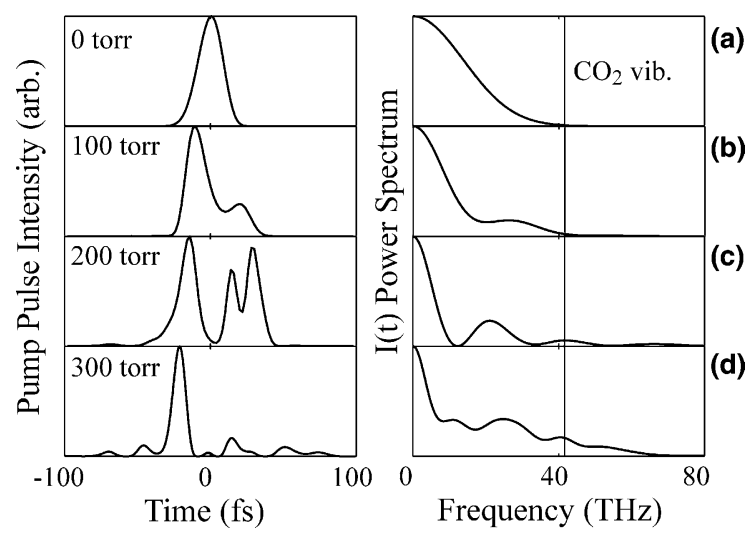

Fig. 2. SHG FROG characterization of the pump pulses exciting the hollow core fiber (left column) and the corresponding power spectrum of the intensity profile (right column) for $\mathrm{CO}_{2}$ pressures of 0 Torr (a), 100 Torr (b), 200 Torr (c), and 300 Torr (d). The appearance of pump pulse power spectral density at high frequencies indicates that the envelope of the pulse is developing faster time structure than the input pulse.

power spectrum (Fig. 2, right) demonstrate that the pump pulse has broken into a quasi pulse train. The evidence suggests that the molecular pulse reshaping mechanisms may be impacting the pulse evolution. Figs. $3 \mathrm{a}-\mathrm{d}$ shows the evolution of the

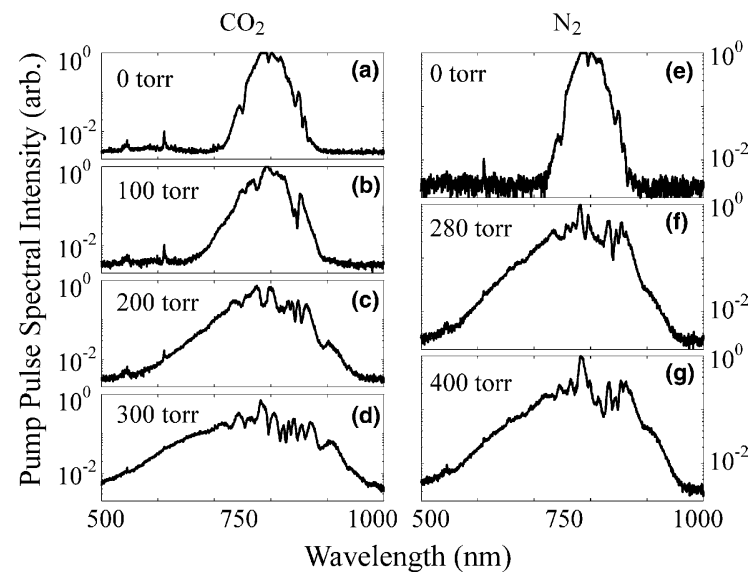

Fig. 3. Spectra for the nonlinear shaped, pump laser pulses exiting the fiber for $\mathrm{CO}_{2}$ at 0 Torr (a), 100 Torr (b), 200 Torr (c), and 300 Torr (d) pressures and for $\mathrm{N}_{2}$ at 0 Torr (e), 280 Torr (f), and 400 Torr (g) pressures. Note the spectra exhibit spectral broadening preferentially on the 'blue' side of the spectrum, indicating self steepening of the pump laser pulses. Furthermore, there is no evidence of any SRS peaks in either the $\mathrm{CO}_{2}$ or $\mathrm{N}_{2}$ data, which supports the assertion that the Raman coherence excitation mechanism is ISRS. 
pulse spectrum for the pump laser pulse shapes shown in Fig. 2. The spectra show an asymmetric broadening at short wavelengths, indicating SS. A detailed study of the ISRS molecular pulse shaping mechanisms will clarify the physics of this pulse evolution.

We also investigated nonlinear propagation in nitrogen gas, which is a diatomic molecule with a fundamental Raman-active vibrational mode at 69.9 $\mathrm{THz}$ (14.3 fs period). Thus, the pump pulse used in this work is too long to excite this mode with traditional ISRS, which is verified by the fact that the pulse envelope power spectrum is diminished by at least 4 orders of magnitude compared with its peak value (see Fig. 4a). As a result, the initial nonlinear pulse evolution of the input pulse is dominated by both SPM and SS. When $\mathrm{N}_{2}$ is placed in the hollow-core fiber at relatively low pressures, the pump pulse propagates through the gas and, as expected, does not excite any vibrational modes, as verified by the absence of Stokes or anti-Stokes peaks in the probe pulse spectrum.

As the $\mathrm{N}_{2}$ pressure in the capillary is increased, we observe a critical pressure at which FM sidebands appear in the probe spectrum, with a mode
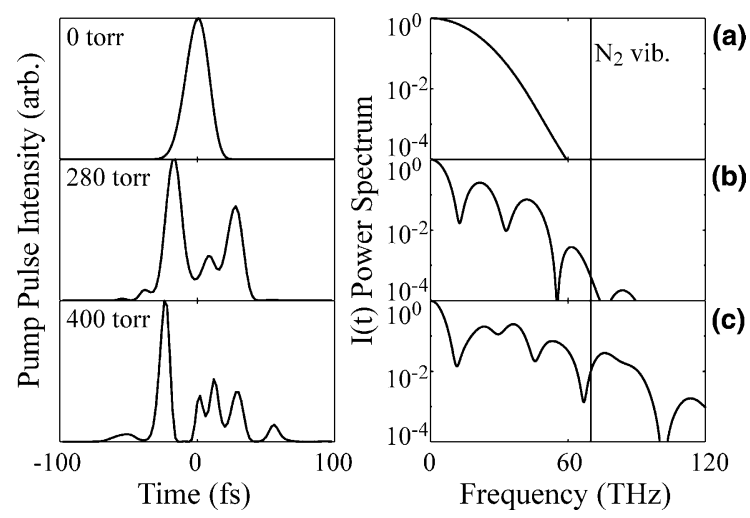

Fig. 4. SHG FROG characterization of the pump pulses exiting the hollow core fiber (left column) and the corresponding power spectrum of the intensity profile (right column) plotted on a $\log$ scale for $\mathrm{N}_{2}$ pressures of 0 Torr (a), 280 Torr (b), and 400 Torr (c) in the hollow core fiber. The pump pulse starts-out too long to excite a vibrational coherence in $\mathrm{N}_{2}$ via ISRS, as evidenced by the lack of power spectral density in the pump $I(t)$ at the $\mathrm{N}_{2}$ vibrational frequency. frequency of $\sim 70 \mathrm{THz}$ (see Figs. 1b, 3, and 4). This is consistent with excitation of a vibrational coherence in $\mathrm{N}_{2}$. The evolution of the pump pulse with pressure as characterized by SHG FROG is shown in Fig. 4. Notice that as the pressure increases, the modified pump pulse acquires a power spectral density component at the $\mathrm{N}_{2}$ vibrational frequency that is absent for the input pulse. The broadened spectra of the pump pulse (Figs. 3e-g) also exhibit preferential broadening on the 'blue' side of the spectrum, and thus experiences self steepening. Fig. 5a shows the sideband intensity at 69.6 THz plotted as a function of $\mathrm{N}_{2}$ gas pressure. At $\sim 350$ Torr we begin to observe a vibrational coherence. Twice the variance (i.e., spectral bandwidth) of the measured pump spectrum is plotted in Fig. 5b, and it is observed to steadily increase with increasing pressure. At sufficiently high pressure, the bandwidth of the pump pulse exceeds that required to access the vibrational period of $\mathrm{N}_{2}$.

Another possible mechanism for the excitation of the vibrational coherence is the buildup of a Stokes field from noise amplified by the pump pulse, referred to as stimulated Raman scattering (SRS) [18]. If this were the case, a Stokes line would appear in the spectrum of the pump pulse at $2 \pi c /\left(\omega_{\mathrm{L}}-\Omega_{\mathrm{v}}\right) \sim 983 \mathrm{~nm}$. The absence of such a Stokes line in the spectrum of the pump pulse (see Figs. $3 \mathrm{e}-\mathrm{g}$ ) indicates that SRS is not the excitation mechanism for this experiment. This is consistent with the fact that our estimate of the peak pump pulse power is $<3 x$ the threshold required for SRS.

The correlation between the pump pulse bandwidth and the probe sideband intensity is shown in Fig. 5c. These data demonstrate that once the bandwidth of the pump laser exceeds the vibrational frequency of $\mathrm{N}_{2}$, the pump pulse is able to excite a vibrational coherence that is consistent with an ISRS mechanism and would be coincidental for SRS. Thus, at a sufficiently high pressure there is an excitation of a Raman coherence of the 14.3 fs vibrational mode in $\mathrm{N}_{2}$ via ISRS.

In conclusion, our work demonstrates that a laser pulse with structure longer than the vibrational period of molecules in which it is propagating can develop structure fast enough to excite a Raman coherence via ISRS due to nonlinear 

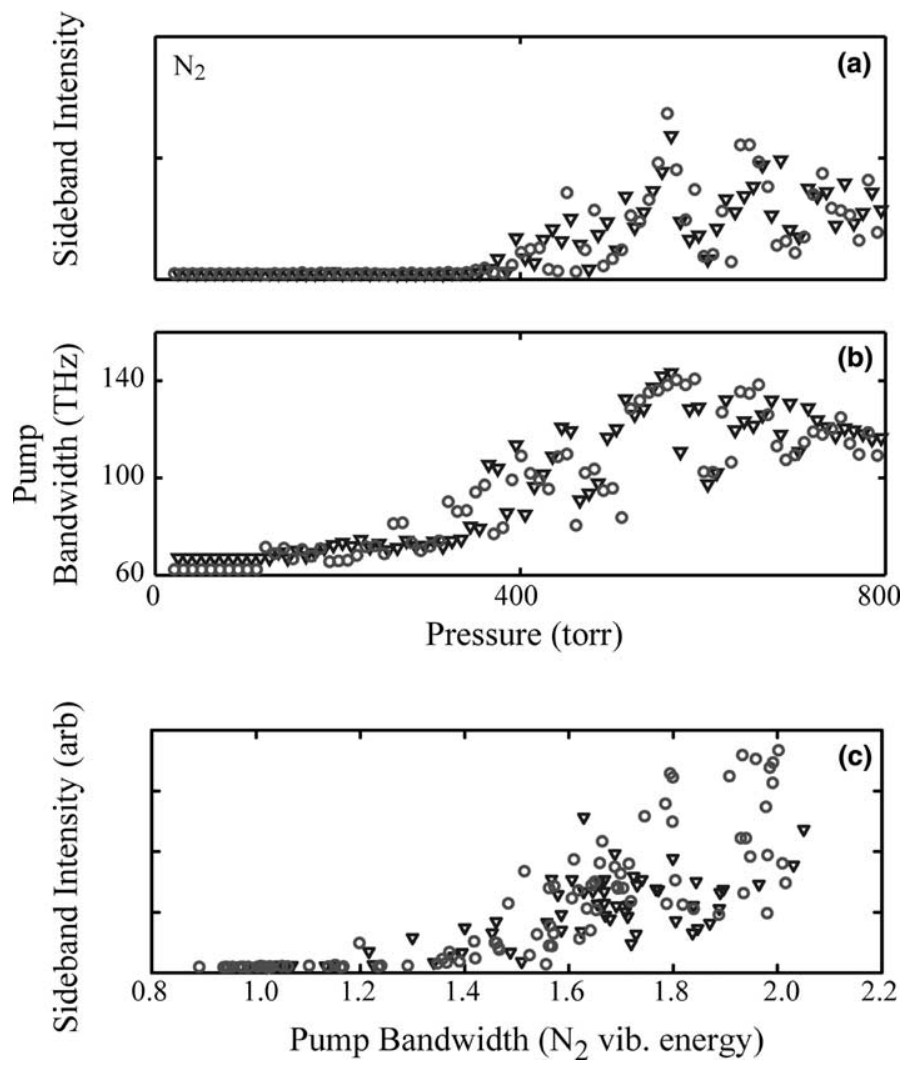

Fig. 5. (a) Scattered sideband intensity at the $\mathrm{N}_{2}$ vibrational frequency. (b) Twice the variance (i.e., bandwidth) of the pump pulse broadened by SPM and ISRS. (c) The intensity of the $\mathrm{N}_{2}$ vibrational sideband correlated with the variance of the pump pulse bandwidth, normalized to the $\mathrm{N}_{2}$ vibrational frequency $(70 \mathrm{THz})$. Clearly, the excitation of the vibrational coherence occurs shortly after sufficient bandwidth is present in the pump pulse. The triangles and circles represent two experimental pressure scans.

pulse shaping. We observe that an intense femtosecond laser pulse propagating in a Raman-active medium can undergo sufficient self-modification to self-seed the ISRS process, allowing the pulse to experience a new nonlinearity. Once this threshold is exceeded, the dynamics of the pulse propagation will change and depend on the balance of other factors acting on the laser pulse. This 'switching on' of new nonlinearities has important implications for the propagation of intense femtosecond pulses in air and optical fibers. Moreover, the self-seeding process may allow concepts of coherent control using ISRS to be extended to a much larger class of molecules with chemically relevant, high-frequency molecular vibrations.

\section{Acknowledgements}

The authors gratefully acknowledge support for this work from the National Science Foundation. We also thank the reviewers for insightful comments that we used to improve the readability of this Letter. In particular, we would like to thank one of the reviewers for suggesting we use the term 'displacive ISRS' to describe the excitation of a Raman coherence by a self-steepened laser pulse.

\section{References}

[1] A.M. Weiner, D.E. Leaird, G.P. Wiederrecht, K.A. Nelson, J. Opt. Soc. Am. B 8 (1991) 1264. 
[2] A.M. Weiner, D.E. Leaird, G.P. Wiederrecht, K.A. Nelson, Science 247 (1990) 1317.

[3] M. Mlejnek, E.M. Wright, J.V. Moloney, Phys. Rev. E 58 (1998) 4903.

[4] M. Mlejnek, M. Kolesik, J.V. Moloney, E.M. Wright, Phys. Rev. Lett. 83 (1999) 2938.

[5] H. Rabitz, R. de Vivie-Riedle, M. Motzkus, K. Kompa, Science 288 (2000) 824.

[6] T.C. Weinacht, R. Bartels, S. Backus, P.H. Bucksbaum, B. Pearson, J.M. Geremia, H. Rabitz, H.C. Kapteyn, M.M. Murnane, Chem. Phys. Lett. 344 (2001) 333.

[7] R.A. Bartels, T.C. Weinacht, S.R. Leone, H.C. Kapteyn, M.M. Murnane, Phys. Rev. Lett. 88 (2002) 033001.

[8] A. Assion, T. Baumert, M. Bergt, T. Brixner, B. Kiefer, V. Seyfried, M. Strehle, G. Gerber, Science 282 (1998) 919.

[9] M. Nisoli, S. De Silvestri, O. Svelto, Appl. Phys. Lett. 68 (1996) 2793.

[10] M. Nisoli, S. de-Silvestri, O. Svelto, R. Szipocs, K. Ferencz, C. Spielmann, S. Sartania, F. Krausz, Opt. Lett. 22 (1997) 522.

[11] C.G. Durfee, S. Backus, M.M. Murnane, H.C. Kapteyn, Opt. Lett. 22 (1997) 1565.
[12] A. Rundquist, C.G. Durfee III, S. Backus, C. Herne, Z. Chang, M.M. Murnane, H.C. Kapteyn, Science 280 (1998) 1412 .

[13] R.A. Bartels, A. Paul, H. Kapteyn, M. Murnane, S. Backus, I. Christov, Y. Liu, D. Attwood, C. Jacobsen, Science 297 (2002) 376.

[14] R.A. Bartels, T.C. Weinacht, N. Wagner, M. Baertchy, C. Greene, M.M. Murnane, H.C. Kapteyn, Phys. Rev. Lett. 88 (2002) 019303.

[15] N. Zhavoronkov, G. Korn, Phys. Rev. Lett. 88 (2002) 203901.

[16] A. Nazarkin, G.K. G, M. Wittmann, T. Elsaesser, Phys. Rev. Lett. 83 (1999) 2560.

[17] Y.R. Shen, N. Bloembergen, Phys. Rev. A 137 (1965) 1787.

[18] G.P. Agrawal, Nonlinear Fiber Optics, Academic Press, San Diego, CA, 2001.

[19] E.T.J. Nibbering, G. Grillon, M.A. Franco, B.S. Prade, A. Mysyrowicz, J. Opt. Soc. Am. B 14 (1997) 650.

[20] D. Homoelle, A.L. Gaeta, Opt. Lett. 25 (2000) 761.

[21] E. Zeek, R. Bartels, M.M. Murnane, H.C. Kapteyn, S. Backus, G. Vdovin, Opt. Lett. 25 (2000) 587.

[22] K.W. DeLong, R. Trebino, J. Hunter, W.E. White, J. Opt. Soc. Am. B 11 (1994) 2206. 